\title{
Case Report: Right Bundle Brunch Block and QTc Prolongation in a Patient with COVID-19 Treated with Hydroxychloroquine
}

\author{
Rosmonaliza Asli, ${ }^{1}$ Muhammad Syafiq Abdullah, ${ }^{1,2}$ Pui Lin Chong, ${ }^{1}$ Dhiya Metussin, ${ }^{1}$ Riamiza Natalie Momin, ${ }^{1}$ Babu Ivan Mani, ${ }^{3}$ \\ and Vui Heng Chong ${ }^{1,2,3 *}$ \\ ${ }^{1}$ Department of Medicine, RIPAS Hospital, Bandar Seri Begawan, Brunei Darussalam; ${ }^{2}$ Institute of Health Sciences, PAPRSB, Universiti Brunei \\ Darussalam, Gadong, Brunei Darussalam; ${ }^{3}$ Department of Medicine, PMMPMHAMB Hospital, Tutong, Brunei Darussalam
}

\begin{abstract}
Novel coronavirus disease (COVID-19) is a highly contagious disease caused by severe acute respiratory distress syndrome coronavirus-2 that has resulted in the current global pandemic. Currently, there is no available treatment proven to be effective against COVID-19, but multiple medications, including hydroxychloroquine (HCQ), are used off label. We report the case of a 60-year-old woman without any cardiac history who developed right bundle brunch block and critically prolonged corrected electrocardiographic QT interval (QTc $631 \mathrm{~ms}$ ) after treatment for 3 days with $\mathrm{HCQ}$, which resolved on discontinuation of the medication. This case highlights a significant and potentially lifethreatening complication of HCQ use.
\end{abstract}

\section{INTRODUCTION}

Novel coronavirus disease (COVID-19) is a highly contagious disease caused by severe acute respiratory distress syndrome coronavirus-2 (SARS-CoV-2) that was identified in December 2019 in China and is now a global pandemic. ${ }^{1}$ Currently, there is no proven effective treatment, and medications proposed to inhibit the virus life cycle such as hydroxychloroquine (HCQ), chloroquine, lopinavir/ritonavir, and remdesivir are used off label. ${ }^{2-5}$ These medications are widely used despite the lack of evidence for their efficacy and safety, and are often used in combination.

\section{CASE REPORT}

A60-year-old woman was admitted to the National Isolation Centre in Brunei after her nasopharyngeal and throat swabs tested positive (reverse transcriptase [RT]-PCR) for severe acute respiratory distress syndrome coronavirus-2 (SARSCoV-2). She was among a group of infected travelers and was linked to a confirmed COVID-19 case through contact tracing. She had just returned from Indonesia 4 days before and developed symptoms (fever, dry cough, weakness, and dyspepsia) on returning. These symptoms had already improved when she was called for testing. Her comorbid conditions included hypertension, hyperlipidemia, and being overweight $\left(31.1 \mathrm{~kg} / \mathrm{m}^{2}\right)$, but she had no known heart disease.

Admission chest radiograph (CXR) was normal, and laboratory investigations revealed mildly elevated C-reactive protein, without lymphopenia (Table 1). She was empirically started on intravenous amoxicillin-clavulanic acid (1.2 g three times daily) and oseltamivir (75 mg twice daily). A repeat CXR on the second day of hospitalization showed bilateral lower zone opacities. As a result, she was transferred to the intensive care unit for close monitoring and was started on lopinavir 400 $\mathrm{mg} /$ ritonavir $100 \mathrm{mg}$ (twice daily). As her condition did not improve, HCQ (400 mg stat dose followed by $200 \mathrm{mg}$ twice daily) was initiated on the fourth day of hospitalization. An electrocardiograph (ECG) on hospital day 4 (before initiation of $\mathrm{HCQ}$ ) was normal, with a corrected QT interval (QTC) of

*Address correspondence to Vui Heng Chong, RIPAS Hospital, Jalan Tutong, Bandar Seri Begawan BA 1710, Brunei Darussalam. E-mail: chongvuih@yahoo.co.uk
$397 \mathrm{~ms}$. Repeat ECGs the following day remained normal (QTc $414 \mathrm{~ms}$ ). The patient's condition deteriorated, requiring intubation and ventilatory support on the fifth day of admission. Blood and urine cultures were negative. Sputum culture isolated Pseudomonas aeruginosa and Serratia marcescens, both sensitive to meropenem. Amoxicillin-clavulanic acid was discontinued, and meropenem (1,000 mg three times daily) was initiated. She was also started on inotropic support. The timeline of events and medications prescribed are shown in Figure 1 and laboratory investigations in Table 1.

On the fifth day, serum troponin I was noted to be mildly elevated. Transthoracic echocardiogram (TTE) showed normal ejection fraction and no regional wall motion abnormalities. Myocarditis secondary to SARS-CoV-2 was considered. Serial monitoring twice daily showed fluctuation of troponin I. On the seventh day of hospitalization, repeat ECG before the morning dose of $\mathrm{HCQ}$ showed a new right bundle branch block (RBBB) and critically prolonged QTc (631 ms) (Figure 2). Hydroxychloroquine was discontinued after a cumulative dose of $1,400 \mathrm{mg}$. Blood investigations on that day showed normal serum $\mathrm{Mg}^{2+}$ and $\mathrm{K}^{+}$but slightly low corrected $\mathrm{Ca}^{2+}$ (Table 1). This was corrected with calcium replacement. A repeat ECG performed 24 hours after the last dose of $\mathrm{HCQ}$ showed normalization of the QTc (433 ms).

On the tenth day of hospitalization, a repeat TTE showed normal cardiac function. She was eventually weaned off inotropes and was extubated on the 14th day of hospitalization. Investigations on the 19th day showed improvement of laboratory parameters apart from elevated D-dimer, and she was otherwise well and had no leg swellings. She was started on low molecular weight heparin. After three consecutive negative RT-PCR results for SARS-CoV-2, she was transferred on the 23rd day of hospitalization to a tertiary hospital, where a computed tomography pulmonary angiogram showed scattered ground-glass opacities consistent with COVID-19 and a small pulmonary embolism on the right. She was started on dabigatran $150 \mathrm{mg}$ twice daily (planned 3 months of treatment) and remained well on follow-up.

\section{DISCUSSION}

Corrected QT interval prolongation is dangerous and can be associated with torsade de pointes, a life-threatening 
TABLE 1

Laboratory results during the course of hospitalization

\begin{tabular}{|c|c|c|c|c|c|c|c|c|c|}
\hline Day of hospitalization & 1 & 5 & 6 & 7 & 8 & 9 & 10 & 19 & 22 \\
\hline \multicolumn{10}{|l|}{ Variable } \\
\hline HB (g/dL) (11.5-15.9) & 11 & 11.0 & 10.2 & 10.5 & 9.8 & 8.4 & 8.8 & 10.5 & 10.8 \\
\hline WCC $\left(\times 10^{9}\right)(4.2-12.6)$ & 6.2 & 5.3 & 7.7 & 12.9 & 12.7 & 11.2 & 10.9 & 9.3 & 6.3 \\
\hline PLT $\left(\times 10^{9}\right)(174-430)$ & 190 & 154 & 155 & 185 & 251 & 246 & 275 & 474 & 429 \\
\hline Lymphocyte $\left(\times 10^{9}\right)$ & 1.2 & 0.9 & 0.9 & 0.4 & 1.5 & 1.3 & 1.4 & 2.1 & 1.7 \\
\hline Neutrophil $\left(\times 10^{9}\right)$ & 4.6 & 4.2 & 6.5 & 12.3 & 10.3 & 9.6 & 9.1 & 6.6 & 3.8 \\
\hline $\mathrm{Na}^{+}(\mathrm{mmol} / \mathrm{L})(136-144)$ & 134 & 134 & 134 & 135 & 140 & 140 & 141 & 139 & 140 \\
\hline $\mathrm{K}^{+}(\mathrm{mmol} / \mathrm{L})(3.5-5.1)$ & 2.7 & 4.0 & 3.8 & 3.7 & 3.8 & 3.7 & 4.3 & 3.5 & 3.6 \\
\hline Cr (umol/L) (39-91) & 73 & 80 & 76 & 75 & 76 & 65 & 67 & 57 & 70 \\
\hline Urea (mmo/L) (2.1-7.1) & 3.3 & 2.8 & 2.6 & 2.8 & 6.0 & 7.1 & 90 & 5.6 & 3.5 \\
\hline $\mathrm{Mg}++(\mathrm{mmol} / \mathrm{dL}(0.66-1.07)$ & - & 0.68 & 0.69 & 0.87 & 0.96 & 0.99 & 0.96 & - & - \\
\hline $\mathrm{Ca}++(\mathrm{mmol} / \mathrm{L})(2.23-2.56)^{\prime}$ & - & 2.13 & 2.17 & $2.14 / 2.25^{\star}$ & $2.14 / 2.45^{\star}$ & 2.33 & 2.47 & - & - \\
\hline Alb (g/L) (35-48) & 36 & 31 & - & 29 & - & - & 20.4 & 29 & 32 \\
\hline $\operatorname{ALT}(\mathrm{U} / \mathrm{L})(<54)$ & 41 & 32 & - & 46 & - & - & 60 & 60 & 62 \\
\hline GGT IU/L) (7-64) & 18 & 16 & - & 29 & - & - & 57 & 51 & 44 \\
\hline ALP (U/L) (38-126) & 54 & 39 & - & 36 & - & - & 59 & 60 & 58 \\
\hline Bilirubin (umol/L) (5.1-20.5) & 9.4 & 20.3 & - & 105.6 & - & - & 50.5 & 23 & 30.2 \\
\hline T protein (g/L) (64-83) & 70 & 67 & - & 64 & - & - & 60 & 63 & 66 \\
\hline $\mathrm{CRP}(\mathrm{mg} / \mathrm{dL})(<0.9)$ & 1.1 & 8.0 & - & - & - & - & 36.8 & - & 0.8 \\
\hline Troponin I (ng/L) $(<30.0)$ & - & 40.3/109.2 & $76.8 / 115.6$ & $66.3 / 46.8$ & - & - & - & - & - \\
\hline Procalcitonin & - & - & - & 0.43 & - & - & - & - & - \\
\hline D-dimer & - & - & - & 506 & - & - & - & 3,189 & 2,149 \\
\hline Blood culture & - & - & - & - & - & - & - & - & - \\
\hline Urine culture & - & - & - & - & - & - & -ve & - & - \\
\hline Sputum culture & - & +ve† & - & - & - & - & - & - & - \\
\hline
\end{tabular}

$\mathrm{HB}=$ hemoglobin; $\mathrm{WBC}=$ white cell count; $\mathrm{PLT}=$ platelets

${ }^{*}$ Calcium levels after intravenous calcium replacement.

† Sputum positive for Pseudomonas aeruginosa and Serratia marcescens.

arrhythmia. Our patient developed RBBB and critically prolonged QTc (QTc > $500 \mathrm{~ms}$ ) after 3 days of HCQ at a cumulative dose of $1,400 \mathrm{mg}$. A systematic review of chronic use of chloroquine and $\mathrm{HCQ}$ in rheumatic conditions reported cardiac side effects to be common. Among patients who were treated with $\mathrm{HCQ}$ who experienced cardiac toxicity ( $n=50$, median duration of use 8 years [range 10 days to 30 years], and cumulative dose of $1,235 \mathrm{~g}$ [range $1.9 \mathrm{~g}-4,380 \mathrm{~g}$ ]), the study reported bundle branch block (26\%), atrioventricular block (24\%), and first- or second-degree heart block (4\%). ${ }^{6}$ Other cardiac adverse effects of $\mathrm{HCQ}$ included ventricular hypertrophy $(32 \%)$, ventricular hypokinesia (16\%), heart failure (ejection fraction $<40 \%$ in $52.9 \%$ ), and valvular dysfunction (8\%), especially with high cumulative doses. ${ }^{6}$ Other adverse effects of HCQ include gastrointestinal, ophthalmic, neurological, musculoskeletal, psychiatric, metabolic, and dermatological abnormalities. ${ }^{7}$

Patients with COVID-19 who require hospitalization are at risk for complications including electrolyte derangements, which are risk factors for QTC prolongation. ${ }^{8}$ Our patient had several risk factors for conduction abnormalities: administration of HCQ, lopinavir/ritonavir, and inotropes, and hypocalcemia. Lopinavir/ritonavir is also associated with the prolongation of QTc, but the ECG after starting this medication was normal. Among the electrolytes associated with the prolongation of QTc, only calcium was slightly low. Inotropes were started 2 days (noradrenaline) and one (dopamine) day before the detection of conduction abnormalities. Unfortunately, we did not obtain an ECG on the third day of HCQ therapy. We considered the possibility of myocarditis. The elevated troponin coincided with the period leading up to conduction abnormalities and peaked several days later, before decreasing. A repeat TTE was normal. We did not perform further investigations for myocarditis, as our patient remained stable. It is possible the other factors discussed contributed to the development of conduction abnormalities, but the resolution of ECGs changes after discontinuation of $\mathrm{HCQ}$ suggested a causal relationship between HCQ and these abnormalities.

Given the lack of proven therapies for COVID-19, the continued use of $\mathrm{HCQ}$ is likely. HCQ and chloroquine have also been used as prophylaxis for COVID-19. ${ }^{7,9}$ With such widespread use, complications can be expected. Combination therapy of chloroquine and azithromycin, both medications associated with QTc prolongation drugs, has recently been advocated. ${ }^{4}$ Our case highlights that significant and lifethreatening conduction abnormalities can occur with the use of HCQ. Therefore, clinicians should exercise caution and assess cardiac risk if considering $\mathrm{HCQ}$ treatment for COVID-19.

Received April 28, 2020. Accepted for publication May 4, 2020.

Published online May 7, 2020.

Acknowledgment: Publication charges for this article were waived due to the ongoing pandemic of COVID-19.

Authors' addresses: Rosmonaliza Asli, Pui Lin Chong, Dhiya Metussin, Riamiza Natalie Momin, Department of Medicine, RIPAS Hospital, Bandar Seri Begawan, Brunei Darussalam, E-mails: rosmonaliza.asli@ moh.gov.bn, lina.chong@moh.gov.bn, ramizah.metussin@moh.gov.bn, and natalie.momin@moh.gov.bn. Muhammad Syafiq Abdullah, Department of Medicine, RIPAS Hospital, Bandar Seri Begawan, Brunei Darussalam, and Institute of Health Sciences, PAPRSB, Universiti Brunei Darussalam, Gadong, Brunei, E-mail: syafiq.abdullah@moh.gov.bn. Babu Ivan Mani, Department of Medicine, PMMPMHAMB Hospital, Jalan Sungai Basong, Tutong, Brunei Darussalam, E-mail: babu.mani@ moh.gov.bn. Vui Heng Chong, Department of Medicine, RIPAS Hospital, 


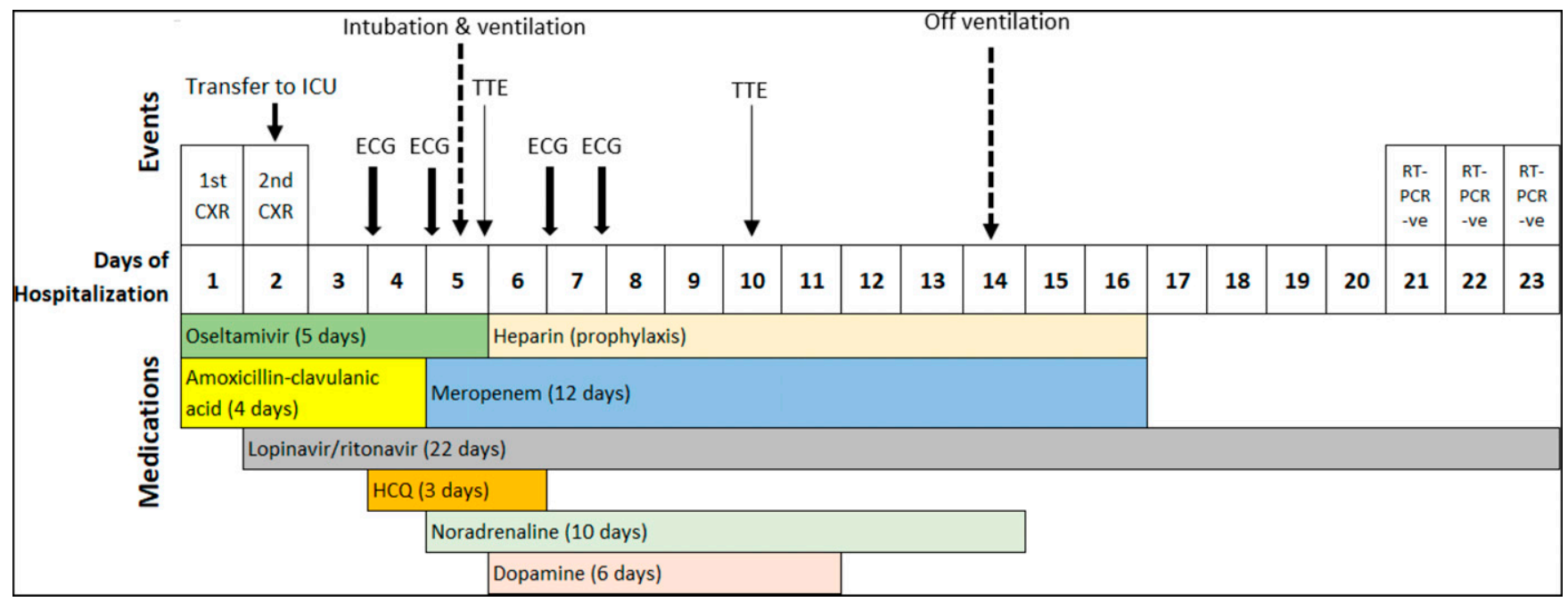

FIGURE 1. Timeline of treatment and events during patient's hospalization (CXR = chest radiograph; $E C G=$ electrocardiograph; $I C U=$ intensive care unit; TTE: transthoracic echocardiogram).

Bandar Seri Begawan, Brunei Darussalam, Institute of Health Sciences, PAPRSB, Universiti Brunei Darussalam, Gadong, Brunei, and Department of Medicine, PMMPMHAMB Hospital, Jalan Sungai Basong, Tutong, Brunei Darussalam, E-mail: chongvuih@yahoo.co.uk.
This is an open-access article distributed under the terms of the Creative Commons Attribution (CC-BY) License, which permits unrestricted use, distribution, and reproduction in any medium, provided the original author and source are credited.

$\begin{array}{llr}\text { Gender. FimAle } & \text { P/PR: } & 92 / 108 \mathrm{~ms} \\ & \text { QRS: } & 210 \mathrm{~ms} \\ & \text { QT/QTC: } & 464 / 631 \mathrm{~ms} \\ & \text { P/QRS } / \mathrm{T} \text { Axis: } & 21 / 84 / 6 \mathrm{deg} \\ & \text { Heart Rate: } & 111 \mathrm{BPM}\end{array}$

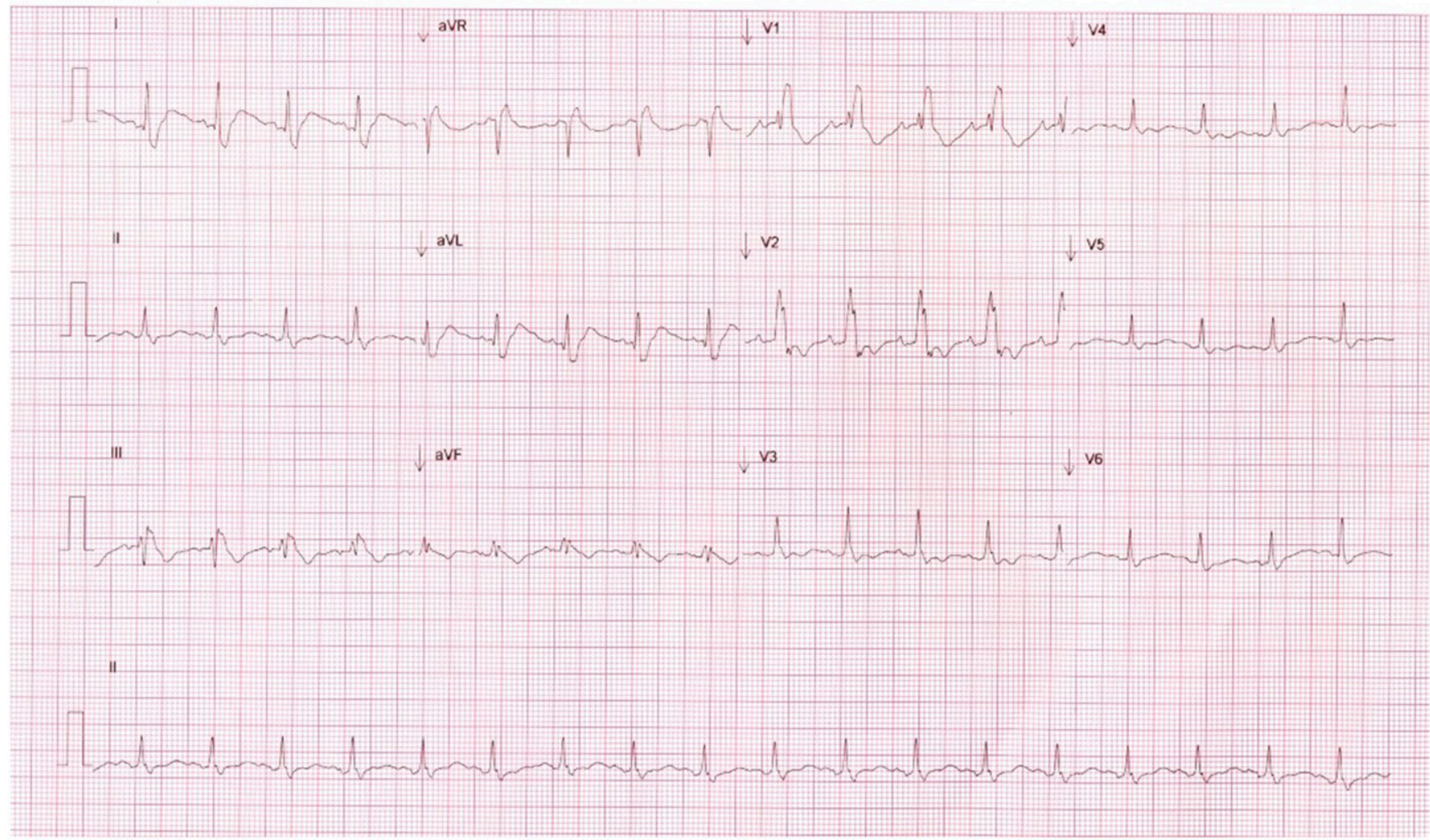

FIGURE 2. Electrocardiograph showing right bundle brunch block (RBBB) and prolonged corrected QT interval on day 4. 


\section{REFERENCES}

1. World Health Organization, 2020. Coronavirus Disease 2019 (COVID-19) Situation Report-51. Geneva, Switzerland: WHO.

2. Kupferschmidt K, Cohen J, 2020. Race to find COVID-19 treatments accelerates. Science 367: 1412-1413.

3. Cao B et al., 2020. A trial of lopinavir-ritonavir in adults hospitalized with severe COVID-19. N Engl J Med. Available at: https://doi.org/ 10.1056/NEJMoa2001282.

4. Gautret $P$ et al., 2020. Hydroxychloroquine and azithromycin as a treatment of COVID-19: results of an open-label nonrandomized clinical trial. Int J Antimicrob Agents. Available at: https://doi.org/10.1016/j.ijantimicag.2020.105949.

5. Zhai P, Ding Y, WuX, Long J, Zhong Y, Li Y, 2020. The epidemiology, diagnosis and treatment of COVID-19. Int $J$ Antimicrob Agents. Available at: https://doi.org/10.1016/j.ijantimicag.2020.105955.
6. Chatre C, Roubille F, Vernhet H, Jorgensen C, Pers YM, 2018. Cardiac complications attributed to chloroquine and hydroxychloroquine: a systematic review of the literature. Drug Saf 41: 919-931.

7. Vandael E, Vandenberk B, Vandenberghe J, Willems R, Foulon V, 2017. Risk factors for QTc-prolongation: systematic review of the evidence. Int J Clin Pharm 39: 16-25.

8. Abena PM et al., 2020. Chloroquine and hydroxychloroquine for the prevention or treatment of novel coronavirus disease (COVID19) in africa: caution for inappropriate off-label use in healthcare settings. Am J Trop Med Hyg. 2020 Apr 22 [ePub ahead of print]. Available at: https://doi.org/10.4269/ajtmh.20-0290.

9. Rathi $S$, Ish $P$, Kalantri $A$, Kalantri S, 2020. Hydroxychloroquine prophylaxis for COVID-19 contacts in India. Lancet Infect Dis S1473-3099: 30313. 